\title{
Postmitotic Prox1 Expression Controls the Final Specification of Cortical VIP Interneuron Subtypes
}

\author{
${ }^{(1)}$ Tevye Jason Stachniak, ${ }^{1,2 *}$ Rahel Kastli, ${ }^{1,2 *}$ () Olivia Hanley, ${ }^{1,2}{ }^{\circledR}$ Ali Özgür Argunsah, ${ }^{1,2}$ \\ Elianne Grietje Theodora van der Valk, ${ }^{1,2}$ George Kanatouris, ${ }^{1,2}$ and Theofanis Karayannis ${ }^{1,2}$ \\ ${ }^{1}$ Laboratory of Neural Circuit Assembly, Brain Research Institute, University of Zurich, CH-8057, Zurich, Switzerland, and ${ }^{2}$ Neuroscience Center \\ Zurich, Zurich, CH-8057, Switzerland
}

Throughout development, neuronal identity is controlled by key transcription factors that determine the unique properties of a cell. During embryogenesis, the transcription factor Proxl regulates VIP-positive cortical interneuron migration, survival, and connectivity. Here, we explore the role of Proxl as a regulator of genetic programs that guide the final specification of VIP interneuron subtypes in early postnatal life. Synaptic in vitro electrophysiology in male and female mice shows that postnatal Prox1 removal differentially affects the dynamics of excitatory inputs onto VIP bipolar and multipolar subtypes. RNA sequencing reveals that one of the downstream targets of Prox1 is the postsynaptic protein Elfn1, a constitutive regulator of presynaptic release probability. Further genetic, pharmacological, and electrophysiological experiments demonstrate that removing Prox1 reduces Elfn1 function in VIP multipolar but not in bipolar cells. Finally, overexpression experiments and analysis of native Elfn1 mRNA expression reveal that Elfn1 levels are differentially controlled at the post-transcriptional stage. Thus, in addition to activity-dependent processes that contribute to the developmental trajectory of VIP cells, genetic programs engaged by Proxl control the final differentiation of multipolar and bipolar subtypes.

Key words: developmental biology; Elfn1; interneuron; Prox1; synapse; VIP

Significance Statement

The transcription factor Proxl generates functional diversification of cortical VIP interneuron subtypes in early postnatal life, thus expanding the inhibitory repertoire of the cortex.

Received May 14, 2021; revised July 23, 2021; accepted July 30, 2021.

Author contributions: T.J.S., R.K., A.O.A., G.K., and T.K. designed research; T.J.S., R.K., O.H., and E.G.T.v.d.V. performed research; T.J.S., R.K., A.O.A., and G.K. analyzed data; T.J.S. and R.K. wrote the first draft of the paper; T.J.S., R.K., 0.H., A.O.A., E.G.T.v.d.V., G.K., and T.K. edited the paper; T.J.S., R.K., and T.K. wrote the paper; A.O.A. contributed unpublished reagents/analytic tools.

The authors declare no competing financial interests.

This work was supported by European Research Council Grant 679175 to T.K.; Swiss National Science Foundation 31003A_170037 to T.K.; Fond zur Förderung des Akademischen Nachwuchs of the UZH Alumni to T.K.; and Swiss Foundation for Excellence in Biomedical Research to R.K. and T.K. 0.H. was supported by an EMBO post-doctoral fellowship. Slidescanner and SP8 confocal imaging was performed with equipment maintained by the Center for Microscopy and Image Analysis, University of Zurich. The AAV Elfn1-GFP virus was created by the Viral Vector Facility at the University of Zurich. We thank Gord Fishell for helpful discussions and for supporting the initiation of this project in his laboratory, while at New York University Medical Center, by providing access to the Prox1fl and Prox1eGFP mice; Leena Ibrahim and Lorenzo Gesuita for comments and efforts on this project; F. Matsuzaki for the generous gift of the conditional Prox1eGFP mouse line (http://www2.clst.riken.jp/arg/mutant\%20mice\%20list.html; (DB0482K); and Catherine Aquino and Lennart Opitz at the Functional Genomics Center Zurich for performing the sequencing of the extracted mRNA and data analysis.

*T.J.S. and R.K. contributed equally to this work.

Correspondence should be addressed to Theofanis Karayannis at karayannis@hifo.uzh.ch.

https://doi.org/10.1523/JNEUROSCI.1021-21.2021

Copyright $\odot 2021$ Stachniak, Kastli et al.

This is an open-access article distributed under the terms of the Creative Commons Attribution 4.0 International license, which permits unrestricted use, distribution and reproduction in any medium provided that the original work is properly attributed.

\section{Introduction}

Although cortical interneuron (IN) diversity begins at their place of birth within distinct embryonic progenitor domains (Mi et al., 2018), single-cell sequencing and manipulation experiments at different developmental stages have suggested that INs undergo their final specification while incorporating into the developing circuit (De Marco García et al., 2011; Mayer et al., 2018). The developmental mechanisms by which distinct types of INs acquire their mature characteristics are only beginning to be revealed (Favuzzi et al., 2019). Interestingly, postmitotic manipulations have demonstrated a persistent requirement for key transcription factors (TFs) in the final specification and maintenance of pyramidal cell fate (De La Rossa et al., 2013). Whether similar TF mechanisms exist for cortical INs remains unknown.

Cortical vasoactive intestinal peptide-expressing (VIP) INs are a diverse population that can be roughly classified into two subgroups: the bipolar and multipolar VIP cells (Bayraktar et al., 1997, 2000; Cauli et al., 2014; Prönneke et al., 2015; Tasic et al., 2018). Although they make up $<5 \%$ of all neurons, VIP INs are critically important for cortical circuit maturation and their malfunction has been implicated in neurodevelopmental disorders (Batista-Brito et al., 2017; Mossner et al., 
2020). The TF Prospero-related homeobox1 (Prox1) is expressed by the majority of INs derived from the caudal ganglionic eminence (Rubin and Kessaris, 2013; Cauli et al., 2014) . Prox1 removal during embryonic development impairs VIP cell migration, cell survival, as well as dendritic development and afferent connectivity of the calretinin-expressing $\left(\mathrm{CR}^{+}\right) \mathrm{VIP}$ bipolar subtype (Miyoshi et al., 2015). Importantly, Prox1 remains expressed in all VIP INs as they incorporate into the developing circuit, suggesting it may have a role in organizing not only their early stages of development, but also their final specification. In addition to requiring Prox1, it has been shown that $\mathrm{CR}^{+}$VIP bipolar cells also depend on proper network activity to acquire their characteristic axo-dendritic profile, as do Reelin- and somatostatin (SST)-expressing INs (De Marco García et al., 2011, 2015; Pan et al., 2018). In contrast, the cholecystokinin-expressing $\left(\mathrm{CCK}^{+}\right)$VIP multipolar subtypes do not show any morphological deficits following activity manipulations (De Marco García et al., 2011), and the effect of Prox1 removal has not been well explored in this cells (Miyoshi et al., 2015). Together, these findings raise the possibility that, during the final developmental steps of VIP IN specification, cell-autonomous and activity-dependent genetic programs work in tandem to guide the network incorporation of VIP subtypes in a differential manner.

In this study, we assess the postnatal requirement of Proxl for the final diversification of VIP IN subtypes. To achieve this, we use a VIPCre driver mouse line combined with a conditional Proxl allele to remove this TF during the first postnatal week (Prox1 postnatal conditional knockout [cKO]) and explore the synaptic properties of bipolar and multipolar VIP cells. Surprisingly, we find that postnatal Proxl removal leads to an alteration of the short-term synaptic dynamics of incoming excitation onto VIP multipolar but not bipolar cells. Using a transcriptomic screen and pharmacological manipulations, we demonstrate that a Proxl-dependent engagement of the trans-synaptic protein Extracellular Leucine Rich Repeat and Fibronectin type III Domain Containing 1 (Elfn1) selectively enables the stronger synaptic facilitation observed in multipolar cells.

\section{Materials and Methods \\ Mice}

All animal experiments were approved by the Cantonal Veterinary Office Zurich and followed Swiss national regulations. Animal lines used in this study are as follows: VIPCre (Vip ${ }^{\mathrm{tml}(\mathrm{cre}) \mathrm{Zjh} / \mathrm{J}}$ ) (Taniguchi et al., 2011), Ail4 (B6;129S6-Gt(ROSA) 26Sortm14(CAG-tdTomato)Hze/J) (Madisen et al., 2010), Proxlfl (Proxl ${ }^{\text {tm2Gco }}$ ) (Harvey et al., 2005), ProxleGFP

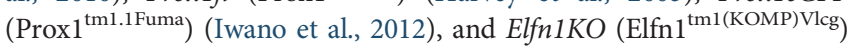
(created by the Knock Out Mouse Project). The following compound lines were created for this study: VIPCre $x$ ProxleGFP and VIPCre $x$ Ai14 $x$ Elfn1KO for staining and electrophysiology experiments; and VIPCre $x$ Ail4 $x$ Proxlfl for mGluR7 staining, in situ, and Elfn1 overexpression experiments. All crosses were set up to produce both $\mathrm{KO}$ and control animals in the same litter (using both males and females), and littermate controls were used throughout the study.

\section{Electrophysiology}

Whole-cell patch-clamp electrophysiological recordings were performed on fluorescently-labeled VIP neurons located in neocortical layers 2 and 3 of barrel cortex (L2/3, approximately bregma -0.5 to $-2.0 \mathrm{~mm}$ ) in acute brain slices prepared from postnatal day 17-34 (P17-P34) male and female mice. Briefly, animals were decapitated, and the brain was dissected out and transferred to cold cutting solution containing the following (in mM): 75 sucrose, $87 \mathrm{NaCl}, 25 \mathrm{NaHCO}_{3}, 25 \mathrm{D}$-glucose, 2.5 $\mathrm{KCl}, 7 \mathrm{MgCl}_{2}$, and $1.25 \mathrm{NaH}_{2} \mathrm{PO}_{4}$, aerated with $95 \% \mathrm{O}_{2} / 5 \% \mathrm{CO}_{2}$; $300 \mu \mathrm{m}$ slices were recovered in ACSF composed of the following (in mM): $128 \mathrm{NaCl}, 3 \mathrm{KCl}, 26 \mathrm{NaHCO}_{3}, 1.25 \mathrm{NaH}_{2} \mathrm{PO}_{4}, 1 \mathrm{MgCl}_{2}, 2 \mathrm{CaCl}_{2}$, and 10 glucose at $34^{\circ} \mathrm{C}$ for $15 \mathrm{~min}$. Acute slices were perfused at a rate of $2-3 \mathrm{ml} / \mathrm{min}$ with oxygenated recording ACSF at room temperature. Patch electrodes were made from borosilicate glass (Harvard Apparatus) and had a resistance of 2-4 $\mathrm{m} \Omega$. The intracellular solution contained the following (in mM): 126 cesium methanesulfonate, $4 \mathrm{CsCl}, 10$ HEPES, 20 phosphocreatine, 4 MgATP, $0.3 \mathrm{NaGTP}, \mathrm{pH}$ 7.3, 290 mOsm, with addition of $2.5 \mathrm{mg} / \mathrm{ml}$ of biocytin.

Experiments were performed in voltage-clamp mode using the Axopatch 200B amplifier (Molecular Devices). Visually guided patch of fluorescently-labeled cells was performed on a Carl Zeiss Axioscope using a Retiga Electro camera (01-ELECTRO-M-14-C-OC, Teledyne Scientific Imaging). Access resistance was monitored to ensure the stability of recording conditions. Recordings with access resistance $>40$ $\mathrm{M} \Omega$, or whole-cell capacitance $<4 \mathrm{pF}$ were excluded. No compensation was made for access resistance, and no correction was made for the junction potential between the pipette and the ACSF. Following a baseline stabilization period (2-3 min), evoked synaptic currents recorded in $2 \mathrm{~min}$ (12 sweeps) at $\mathrm{V}_{\mathrm{h}}=-70 \mathrm{mV}$ were averaged and analyzed using Clampfit10 (Molecular Devices). Two electrical stimuli from a Digitimer isolated stimulator (DS2A Mk.II) were delivered at $50 \mathrm{~Hz}$ through a monopolar glass pipette (2-4 $\mathrm{M} \Omega$ ) positioned in L2/3, close to the soma or proximal dendrites of the recorded cells. The stimulating electrode was placed typically 50$150 \mu \mathrm{m}$ from the recorded cell, parallel to the pial surface. Stimulation intensity and duration were adjusted to produce stable evoked EPSC amplitudes. Stimulation intensities were larger for bipolar cells $(86 \pm 1 \mathrm{~V}$ for $103 \pm 6 \mu \mathrm{s})$ than for multipolar cells $\left(74 \pm 2 \mathrm{~V}\right.$ for $86 \pm 6 \mu \mathrm{s}, p=3 \times 10^{-6}, p=0.06$ ) but did not differ between genotypes (multipolar Proxl control: $69 \pm 3 \mathrm{~V}$ for $57 \pm 5$ $\mu \mathrm{s}, \mathrm{cKO}: 71 \pm 4 \mathrm{~V}$ for $74 \pm 10 \mu \mathrm{s}, p=0.6, p=0.2$; bipolar Prox 1 control: $85 \pm 2 \mathrm{~V}$ for $91 \pm 9 \mu \mathrm{s}, \mathrm{cKO}: 84 \pm 3 \mathrm{~V}$ for $88 \pm 15 \mu \mathrm{s}, p=0.9, p$ $=0.9$; multipolar Elfn 1 control: $77 \pm 3 \mathrm{~V}$ for $114 \pm 13 \mu \mathrm{s}$, Het: $82 \pm 3 \mathrm{~V}$ for $125 \pm 19 \mu \mathrm{s}, p=0.2, p=0.6$; bipolar Elfn 1 control: $86 \pm 3 \mathrm{~V}$ for $113 \pm 13 \mu \mathrm{s}$, Het: $89 \pm 2 \mathrm{~V}$ for $130 \pm 15 \mu \mathrm{s}, p=0.3, p=$ $0.4, t$ test). For comparison between L2/3 and L4 stimulation, the stimulating electrode was moved from the initial position in $\mathrm{L} 2 / 3$ to L4, parallel to the bipolar cell dendrites. Stimulation intensity and duration were held constant ( $61 \pm 4 \mathrm{~V}$ for $63 \pm 5 \mu \mathrm{s})$. (RS)- $\alpha$-methylserine-O-phosphate (MSOP; $100 \mu \mathrm{M}, \mathrm{pH}$ 7.4) was dissolved in water and applied in the bath.

\section{Cell sorting and RNA sequencing}

eGFP-labeled cells were purified from the P12 cortices of control and cKO Prox1 animals (VIPCre $x$ ProxleGFP/+ and VIPCre $x$ ProxleGFP/ $e G F P$ ) as described previously (Hempel et al., 2007). Briefly, animals were anesthetized in $2 \% \mathrm{v} / \mathrm{v}$ isoflurane $(\sim 1.5 \mathrm{~min})$, decapitated, the brain was extracted, the olfactory bulb and cerebellum were removed, and the brain was cut into $400-\mu \mathrm{m}$-thick coronal sections using a vibratome (Leica Microsystems, VT1000S; speed 5, vibration frequency 7-8), while in bubbling ice-cold ACSF (recipe as above, with $1 \mathrm{~mm} \mathrm{CaCl}$ and $1 \mathrm{~mm} \mathrm{MgCl}_{2}$ ) in the chamber. Sections were collected and transferred into a protease digestion solution of ACSF (with Pronase, $1 \mathrm{mg} \mathrm{ml}^{-1}$ ), for $25 \mathrm{~min}$ and then transferred into a quenching solution of ACSF (with 1\% FBS). Microdissection of the cortex to include the somatosensory areas was performed using a fluorescent dissection microscope (Olympus, MVX 10). Dissected cortices were collected in a $15 \mathrm{ml}$ Falcon tube containing $1.5 \mathrm{ml}$ of ACSF sorting solution containing 1\% FBS and DNase, and cells were dissociated by gently triturating 10 times with a large, then medium, and finally small fire-polished Pasteur pipette while avoiding the generation of bubbles. The cell suspension was 
then passed through a $50 \mu \mathrm{m}$ filter (Sysmex CellTrics) before automated FACS using the MoFlo or FACSAria devices. A GFP-negative littermate control cortex was also included as a negative control for the FACS setup. Cells were collected into Arcturus Picopure extraction buffer and immediately processed for RNA isolation using the Arcturus PicoPure Isolation Kit (KIT0204). RNA quality and quantity were measured using Agilent High Sensitivity RNA ScreenTape system (High Sensitivity RNA Screen Tape 5067-5579-5580-5581). All samples had high-quality scores between 6 and 8 RIN. Four or five RNA samples of each genotype were used to prepare 19 barcoded libraries.

The libraries were prepared using the Smart-seq 2 protocol (Picelli et al., 2013). Briefly, total RNA was placed in $4 \mu \mathrm{l}$ of lysis buffer $(0.1 \% \mathrm{v} / \mathrm{v}$ Triton X-100, 2.5 mm dNTPs, $2.5 \mu$ m oligo-dT, 1 U/ $\mu$ l Promega Rnasin Plus RNase inhibitor). Reverse transcription was performed followed by cDNA amplification. The quality of the cDNAs was evaluated using an Agilent 2100 Bioanalyzer. The resulting cDNA (1 ng) was fragmented using Illumina Nextera XT according to standard protocol. Nextera adaptors containing Unique Dual Indices were added by PCR. The libraries were double-sided size, selected, and quantified using an Agilent 4200 TapeStation System.

TruSeq SR Cluster Kit HS4000 (Illumina) was used for cluster generation using $10 \mathrm{pm}$ of pooled normalized libraries on the cBOT. Sequencing was performed on the Illumina HiSeq 4000 single end 100 bp, using the TruSeq SBS Kit HS4000 (Illumina).

\section{Proxl stainings}

VIPCre $x$ ProxleGFP (Control and cKO) animals age $\mathrm{P} 4$ and $\mathrm{P} 6$ were deeply anesthetized before transcardial perfusion with first $1 \times$ PBS followed by ice-cold $4 \%$ PFA. The brains were dissected and postfixed in ice-cold $4 \%$ PFA for $1 \mathrm{~h}$, then cryo-protected in a $30 \%$ sucrose solution at $4^{\circ} \mathrm{C}$ for $>24 \mathrm{~h}$. The brains were embedded in OCT using a peel-away mold and stored at $-80^{\circ} \mathrm{C}$. Coronal $20-\mu \mathrm{m}$-think brain sections containing barrel cortex were cut and collected on-slide using a cryostat (Microm International, HM $560 \mathrm{M}$ ), and the slides were stored at $-80^{\circ} \mathrm{C}$ until further processing.

The slides were thawed and washed using $1 \times$ PBS $(3 \times 5 \mathrm{~min})$ before being blocked using 1.5\% normal donkey serum (NDS) and $0.05 \%$ Triton X-100 in $1 \times$ PBS for $1 \mathrm{~h}$. The primary antibodies GFP (chicken anti GFP, Abcam, ab13970) and Prox1 (goat anti Prox1, R\&D Systems, AF2727) were both diluted 1:1000 in the blocking solution and left to incubate overnight at $4^{\circ} \mathrm{C}$. The next day, the slides were washed $(3 \times$ $7 \mathrm{~min}$ ) with $1 \times$ PBS before applying the secondary antibodies (donkey anti-goat 650, Thermo Fisher Scientific, SA5-10089 and donkey antichicken, Jackson ImmunoResearch Laboratories, 703-545-155) diluted at $1: 1000$ in $1 \times$ PBS for $2 \mathrm{~h}$. The slides were coverslipped with Fluoromount-G with DAPI (00-4959-52) and imaged using an inverted Leica Microsystems SP8 microscope.

$m$ GluR7 staining

VIPCre $x$ Ail4 x Proxlfl (Control and cKO) animals aged P21 were perfused, postfixed, and cut at $20 \mu \mathrm{m}$ as described above. Cryosections were washed in $0.3 \%$ Triton X-100 in $1 \times$ PBS $(3 \times 5 \mathrm{~min})$ and blocked for $2 \mathrm{~h}$ using $5 \%$ BSA, $10 \%$ NDS, $0.3 \%$ Triton X-100 in $1 \times$ PBS. The primary antibodies mGluR7 (rabbit anti mGluR7, Millipore, 07-239) and tdTomato (goat anti-tdTomato, SICGEN Antibodies, AB8181-200) were diluted at 1:500 and 1:1000 in 1\% BSA, 5\% NDS, 0.3\% Triton X-100 in $1 \times$ PBS and applied to the slide overnight at $4^{\circ} \mathrm{C}$. The next day, the slides were washed $(4 \times 7 \mathrm{~min})$ with $1 \times$ PBS before applying the secondary antibody solution containing donkey anti-rabbit 488 (Thermo Fisher Scientific, A21206) and donkey anti-goat Cy3 (Jackson ImmunoResearch Laboratories, 705-165-147) diluted at 1:1000 in the same solution as the primary antibodies and left to incubate for $2 \mathrm{~h}$ at room temperature. They were then coverslipped using Vectashield with DAPI (H-1200-10) and stored at $4^{\circ} \mathrm{C}$ until imaging.
Images were taken on a Leica Microsystems SP8 inverted microscope, with a $63 \times$ oil immersion objective at $1 \times$ zoom. Upper L2/3 and L4 were identified using the DAPI signal, and images sized $1600 \times 1600$ pixels were taken in both locations with voxel size $0.1154 \times 0.1154$ $\times 0.297 \mu \mathrm{m}(X Y Z)$.

\section{mRNA ISH experiments}

VIPCre x Ai14 x Proxlfl animals aged P12 were perfused, postfixed, and cut at $20 \mu \mathrm{m}$ as described above. ISH for Elfn1 mRNA and CR (Calb2) mRNA was performed using the RNAscope kit (RNAscope Intro Pack for Multiplex Fluorescent Reagent Kit v2- Mm, 323136). Briefly, the slides were thawed, and OCT residue was removed using $1 \times$ PBS (3$\times 5 \mathrm{~min}$ washes). The slides were then baked for $30 \mathrm{~min}$ at $60^{\circ} \mathrm{C}$, postfixed for $30 \mathrm{~min}$ in 4\% PFA, dehydrated in an Ethanol dilution series (50\%, 70\%, $2 \times 100 \%)$, and incubated with RNAscope Hydrogen Peroxide for $10 \mathrm{~min}$. RNAscope $1 \times$ Target Retrieval Reagents was brought to the boil, and the slides were submerged for 2 min. Protease III was added to the slides and left to incubate for $45 \mathrm{~min}$ at $40^{\circ} \mathrm{C}$. After the pretreatment, the probes (CR: Mm-Calb2, 313641 and Elfn1: MnElfn1, 449661-C3) were hybridized to the slices for $2 \mathrm{~h}$ at $40^{\circ} \mathrm{C}$, and the signal was amplified using branched DNA amplification methods and visualized with Opal dyes (Opal 520 FP1487001KT and Opal690 FP1497001KT).

Following the RNAscope protocol, the slices were immunostained to retrieve the tdTomato signal that was lost during the RNAscope protocol. Slides were washed 2 times with $1 \times$ PBS for 5 min before being blocked for 30 min using 10\% NDS and 1\% BSA in $1 \times$ PBS. The primary antibody against tdTomato (goat anti tdTomato, SICGEN Antibodies, AB8181-200) was diluted 1:700 in $1 \times$ PBS $1 \%$ BSA and left to incubate at $4^{\circ} \mathrm{C}$ overnight followed by a $2 \mathrm{~h}$ incubation of secondary antibody at room temperature (donkey anti-goat Cy3, Jackson ImmunoResearch Laboratories, 705-165147, diluted 1:1000 in $1 \times$ PBS $1 \%$ BSA). Slides were coverslipped with Fluoromount-G with DAPI (00-4959-52) and imaged using a Slidescanner (Carl Zeiss, AxioScan Z1). Mosaic images of the whole cortex were taken using a $20 \times$ objective.

\section{Elfn1 overexpression/rescue experiments}

To induce cell type-selective Elfn1 overexpression, a custom AAV plasmid was created using a three-way ligation of the $4.4 \mathrm{~kb} \mathrm{KpnI} / \mathrm{BsrGI}-$ fragment of the Addgene \#28305 plasmid (backbone-ITRs-Promoterdlox sites), the $0.8 \mathrm{~kb}$ BsrGI/BamHI-fragment of the Addgene \#85225 plasmid (2A-EGFP), and a $2.5 \mathrm{~kb}$ PCRfragment from an Elfn1-GFP plasmid (Elfn1 sequence) (Sylwestrak and Ghosh, 2012). The custom virus is available on request from the viral vector facility at the University of Zurich (https://www.vvf.uzh.ch/).

VIPCre $x$ Ail4 $x$ Proxlfl control and cKO pups were injected with the Elfn1-AAV construct at P4. They were anesthetized using isoflurane, and the injection was performed with a glass micropipette attached to a Nanolitre 2010 pressure injection apparatus (World Precision Instruments). The micropipette was inserted into the barrel field, and a total of $210 \mathrm{nl}(70 \mathrm{nl}$ each) of virus was injected at three different depths $(300,200$, and $100 \mu \mathrm{m})$. The pups were then placed back in their home-cage and monitored for $4 \mathrm{~d}$. Two to 4 weeks after the injection, they were killed and used for electrophysiology recordings as described above.

\section{Experimental design and statistical analysis}

Electrophysiology data analysis. Values are represented as mean \pm SEM. Number of measurements $(n / N)$ indicates cells recorded $(n)$ from animals $(N)$, typically using 1 cell per slice to recover biocytin-stained cell morphology. Cell type was classified as bipolar versus multipolar based on cell body morphology (ovoid vs round) and number and orientation of dendritic processes emanating from it ( 2 or 3 dendrites perpendicular to pia (for bipolar) versus $\geq 3$ processes in diverse orientations 
(for multipolar) (for a more comprehensive description, see also Cauli et al., 2014). In addition, the layer localization of the two populations differed, with multipolar cells found primarily in the upper L2, compared with the bipolar cells found throughout L2/3. Classification used both the initial determination, before patching fluorescent-labeled cells, and post hoc verification of recovered biocytin-labeled cells. Matched recordings were performed with Proxl control and Proxl cKO littermates on the same day, whenever possible. Elfn1 rescue experiments included control recordings from uninfected neighboring cells in tissue transfected with Elfn1-GFP virus. Elfn1-GFP transfection of patched cells was verified by measuring green fluorescence intensity (average fluorescence intensity $>6000$ a.u.). Statistical testing was done in MATLAB. Comparisons within conditions were made by two-tailed paired Student's $t$ test, treatment versus baseline. Comparisons across conditions or between genotypes were done with an unpaired $t$ test assuming unequal variance. For multiple comparisons, a one-way or two-way ANOVA was done with a Bonferroni post hoc test.

RNA sequencing data analysis. The raw sequence reads were first cleaned by removing adapter sequences, trimming low-quality ends, and filtering reads with low quality (phred quality $<20$ ) using Trimmomatic (version 0.33) (Bolger et al., 2014). The read alignment was done with STAR (version 2.5.3a) (Dobin et al., 2013) As a reference, we used the Ensembl genome build GRCm38 with the gene annotations downloaded on 2015-06-25 from Ensembl. The STAR alignment options were as follows: “-outFilterType BySJout -outFilterMatchNmin 30 -outFilter MismatchNmax 10 -outFilterMismatchNoverLmax 0.05 -alignSJD BoverhangMin 1 -alignSJoverhangMin 8 -alignIntronMax 1000000 alignMatesGapMax 1000000 -outFilter MultimapNmax 50."

Gene expression values were computed with the function featureCounts from the R package Rsubread (version 1.26.0) (Liao et al., 2013). The options for featureCounts were as follows: min mapping quality 10 - min feature overlap $10 \mathrm{bp}$ - count multimapping reads count only primary alignments - count reads also if they overlap multiple genes. One sample was excluded from further analysis based on quality control standards (see Fig. 2B).

To detect differentially expressed genes, we applied a count-based negative binomial model implemented in the software package EdgeR ( $R$ version: 3.6.0, EdgeR version: 3.26.1) (Robinson et al., 2010). The differential expression was assessed using an exact test adapted for overdispersed data. Genes showing altered expression with adjusted (Benjamini and Hochberg method) $p$ value $<0.05$ were considered differentially expressed.

A list of potential candidate genes was generated by selecting genes that had a $\log 2$ ratio $\geq|0.5|$ and $p$ value $\leq 0.01$ (see Extended Data Fig. 21). This list was analyzed for Gene Ontology (GO) enrichment using g: Profiler (Raudvere et al., 2019) (https://biit.cs.ut.ee/gprofiler/gost).

mGluR7 staining data analysis. A customized MATLAB script was used to analyze 3D mGluR7 colocalizations on VIP-tdTomato-positive processes. First, intensity histograms of tdTomato signal (channel 1) and mGluR7 signal (channel 2) were normalized to a corresponding reference image. Next, cell somas were removed from the tdTomato channel according to the following pipeline. First, a blob enhancement filter was used by convolving tdTomato channel with a Gaussian kernel to increase the signal-to-noise ratio. After, tdTomato-positive cell bodies were segmented using Otsu thresholding. Active contours were then used to finalize the segmentation. Finally, the masked tdTomato-positive somas were removed from the dataset, leaving exclusively tdTomato-positive VIP processes in channel 1. Otsu thresholding was performed on the histogram-matched channel 2 to segment mGluR7 puncta. Last, $3 \mathrm{D}$ masked stacks for channels 1 and 2 were combined with a logical AND operator and 3D connected components analysis was used to count the putative mGluR7 puncta on VIP processes. The number of colocalizations was normalized by the volume of the processes.
mRNA ISH data analysis. Image analysis was performed using custom-written MATLAB codes. A two-dimensional difference of Gaussian feature enhancement algorithm was used to improve the VIP-tdTomato images, followed by Otsu thresholding to get an initial segmentation of neuronal cell bodies. To ensure an accurate representation of the cell body, segmentation of VIP INs was finalized using active contour segmentation (Kass et al., 1988).

During preprocessing of the $C R$ and Elfn1 images, background subtraction was performed using a disk-shaped structuring. Subsequently, fluorescent intensity levels of both $C R$ and Elfn1 were measured within each of the segmented VIP IN cell bodies. The somatosensory region, as well as L1-L6, were segmented manually using the DAPI channel as reference (see Fig. $4 A, B$ ). To define whether a VIP cell is $\mathrm{CR}^{+}$, we followed a Bayesian approach by assuming $80 \%$ of VIP cells are $\mathrm{CR}^{+}$(Kubota et al., 2011). Hence, once mean $C R m R N A$ intensity per cell was calculated for each image, the population was thresholded such that $\sim 80 \%$ of total VIP cells are considered $\mathrm{CR}^{+}$. Statistical analysis of the data was done using the Mann-Whitney $U$ test.

\section{Data and code availability}

Data and custom-written codes are available on request. The RNAseq data have been uploaded to the ENA database (accession number PRJEB37790).

\section{Results}

\section{Postnatal Prox1 removal leads to changes in short-term dynamics of incoming excitatory events onto VIP multipolar but not bipolar cells}

Cell-specific wiring properties are a prominent feature of IN cell type diversity. Previous research found that embryonic Proxl removal leads to aberrant network integration of VIP bipolar cells (Miyoshi et al., 2015), whereas the effect on multipolar cells remained unknown. We therefore wanted to examine the effect of postnatal loss of function of Prox $1(\mathrm{cKO})$ on the synaptic properties of bipolar as well as multipolar VIP cells. To remove Prox1 starting from around P3 onwards, we used a VIP knock-in mouse line that drives the postnatal expression of Cre from the endogenous peptide locus (VIPCre) and combined it with a conditional Prox 1 allele. The Prox 1 Exon2 is flanked by loxp sites and recombination shifts eGFP into frame (ProxleGFP) to label all Prox1-expressing VIP cells (Fig. 1A,B).

To examine the timeline of Prox 1 removal, we collected and sectioned brains from $\mathrm{cKO}$ and control VIPCre $x$ ProxleGFP pups at ages $\mathrm{P} 4$ and $\mathrm{P} 6$ and stained them for GFP and Prox1. At P4, the eGFP signal was weak, but it increased markedly by P6 (Fig. $1 C$ ), indicating an accumulation of eGFP protein within those $2 \mathrm{~d}$. In line with this result, we observed that at P6 nearly all of the bright eGFPexpressing cKO cells show a complete absence of Prox 1 signal, while the majority of control cells display Prox1 signal (Fig. 1C). These results show that the removal of Prox 1 starts right before $\mathrm{P} 4$ and is largely completed by P6.

To assess the involvement of Prox 1 in postnatal synaptic properties of VIP subtypes, we examined the short-term dynamics of evoked synaptic responses onto control and Prox1 cKO cells at P17-P21 (Fig. 1B). We calculated the paired-pulse ratio (PPR), which provides a stable measure of whether presynaptic release probability for a given synapse is low (PPR $>1)$, moderate (PPR $\sim 1)$, or high $(\mathrm{PPR}<1)$. We found that glutamatergic synapses onto control VIP bipolar cells show negligible synaptic facilitation (mean PPR: $1.15 \pm 0.13$ ) and that Prox1 cKO does not affect 
A
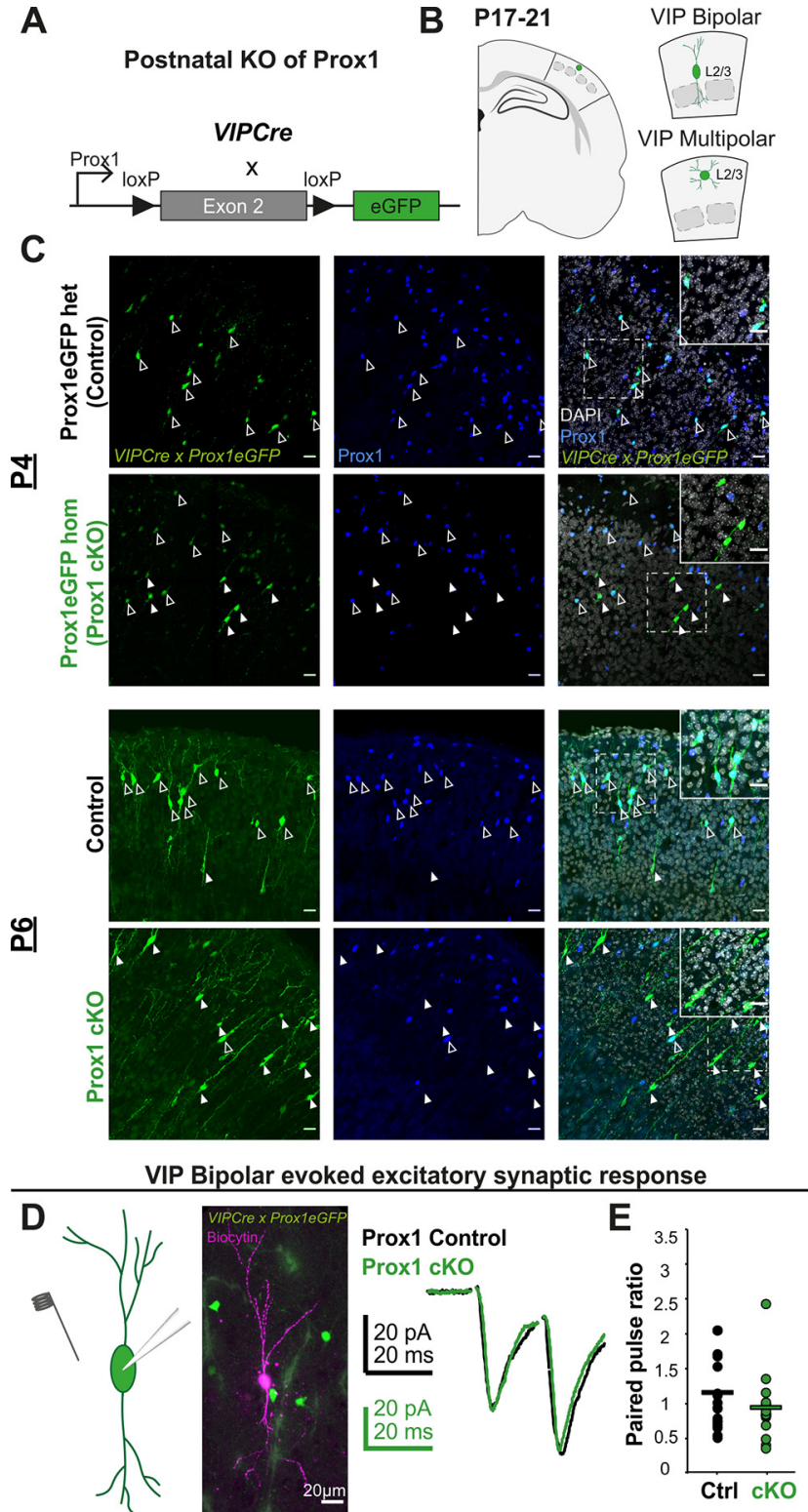

VIP Multipolar evoked excitatory synaptic responses
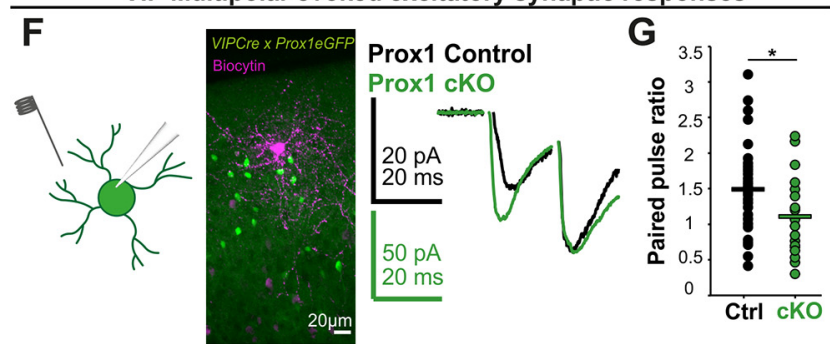

Figure 1. Postnatal Prox1 removal leads to changes in short-term dynamics of incoming excitatory events onto VIP multipolar but not bipolar cells. $\boldsymbol{A}$, Visual representation of Prox1 CKO strategy. VIPCre turns on postnatally and removes part of the coding region of the Prox1 locus, shifting eGFP into frame and allowing for the visualization of the control (Prox1 het) and Prox1 cK0 cells. $\boldsymbol{B}$, Schematic representation of the experiment. L2/3 multipolar and bipolar VIP cells in the somatosensory barrel cortex were stained for Prox1 at P4 and P6 (C) and recorded at P17-21 in acute brain slices from control and Prox1 CK0 animals (D). C, Images of Prox1 staining in L2/3 VIPCre x Prox1eGFP cells at P4 (top) and P6 (bottom) in control and Prox1 cK0 tissue. At P4, recombination of the Prox1 allele has already begun, and many strongly eGFP-positive cells show no Prox1 labeling. At P6, almost all VIPCre cells are strongly fluorescent, and recombination of the allele is complete as shown by the lack of Prox1 expression in the fate-mapped cells. Open arrows highlight VIP-positive, Prox1-positive cells. Filled arrows indicate VIP-positive, Prox1-negative cells. Insets, Close-up of the dashed- the PPR significantly (mean PPR $0.93 \pm 0.15$ ) (Fig. 1D,E). On the other hand, excitatory inputs onto VIP multipolar cells show more pronounced facilitation in the control condition (mean PPR: $1.51 \pm 0.11$ ) and a notable reduction of the PPR on removal of Prox1 (mean PPR: $1.13 \pm 0.1$ ) (Fig. $1 F, G)$. These results demonstrate that, in control conditions, facilitation of glutamatergic synapses onto VIP multipolar cells is higher than onto bipolar cells. Previous reports using paired recordings in the cortex of juvenile or adult animals confirm that $\sim 80 \%$ of pyramidal to bipolar synapses display high release probability with synaptic depression at $18 \mathrm{~Hz}$ and that synapses onto $\mathrm{L} 2 / 3 \mathrm{CR}^{+}$bipolar cells, on average, display short-term synaptic depression at $10 \mathrm{~Hz}$, with no change at $50 \mathrm{~Hz}$ (Porter et al., 1998; Caputi et al., 2009) (https://portal.brain-map.org/explore/ connectivity/synaptic-physiology). Importantly, our data also show that postnatal removal of Proxl leads to a decrease in the facilitation of excitatory synapses onto multipolar, but not bipolar, VIP cells.

Loss of Prox1 leads to a downregulation of the trans-synaptic protein Elfn1

Having identified a Prox1-dependent enhancement of synaptic facilitation onto multipolar cells, we hypothesized that Prox1 transcriptional regulation might impact genes that encode synaptic proteins. To identify such potential downstream targets of Proxl, we performed an RNA sequencing screen on control and Prox1 cKO cells, after FACS of the GFP ${ }^{+}$VIP cells at P12 (Fig. $2 A, B)$. Differential gene expression analysis on the data identified several upregulated and downregulated candidate genes (Fig. 2C, $D$; see also Extended Data Fig. 2-1), which were analyzed for GO enrichment. The GO analysis revealed that most of the upregulated genes in Proxl cKO cells are associated with glial cell programs (Fig. 2F). This result suggests that, in control VIP cells, Prox1 suppresses those glial cell programs postnatally, directing the cell toward a neuronal fate instead. In line with this, the most enriched downregulated genes are associated with synapses and synapse-associated signaling (Fig. 2F,G). Within those synapseassociated processes, we specifically looked for genes that could mediate trans-synaptic interactions between the postsynaptic VIP and the presynaptic excitatory cell to regulate synaptic facilitation. One such gene was the Elfn1 (Fig. 2D), which is responsible for the strongly facilitating excitatory synaptic inputs onto SST INs (Sylwestrak and Ghosh, 2012). Postsynaptic Elfn1 produces cell-autonomous suppression of presynaptic glutamate release through its trans-synaptic recruitment of metabotropicglutamate receptor 7 (mGluR7) (Tomioka et al., 2014; Stachniak et al., 2019).

The RNA sequencing data showed that the level of Elfn 1 mRNA in VIP cells is high at P12 (Fig. 2E). To assess

$\leftarrow$

line boxes. $\boldsymbol{D}, \boldsymbol{F}$, Left, Schematic representation for probing the evoked excitatory synaptic responses onto bipolar and multipolar Prox1 control and CKO VIP cells. The stimulating electrode is shown close to the soma and proximal dendrites of the eGFP-positive cells, which are recorded in whole-cell patch-clamp mode. Middle, The recorded cells were filled with biocytin, and their morphology revealed post hoc. Right, Examples of a pair of evoked excitatory synaptic responses for control and cKO cells, which are overlaid and scaled to the second response. $\boldsymbol{E}$, All data points for PPR (second/first response) for control $(n / N=18 / 11)$ and $c K 0$ $(n / N=13 / 11)$ bipolar VIP cells $(p=0.3, t=1.1)$. G, All data points for PPR of control $(n / N=33 / 18)$ and $c K 0(n / N=19 / 14)$ multipolar VIP cells $(p=0.03, t=2.17)$. Statistics: $t$ test. ${ }^{*} p<0.05$. 
A

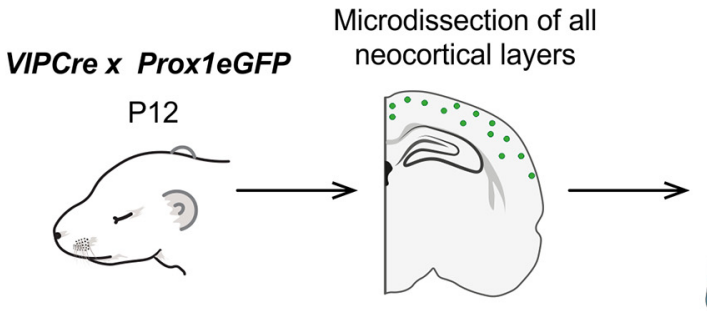

C

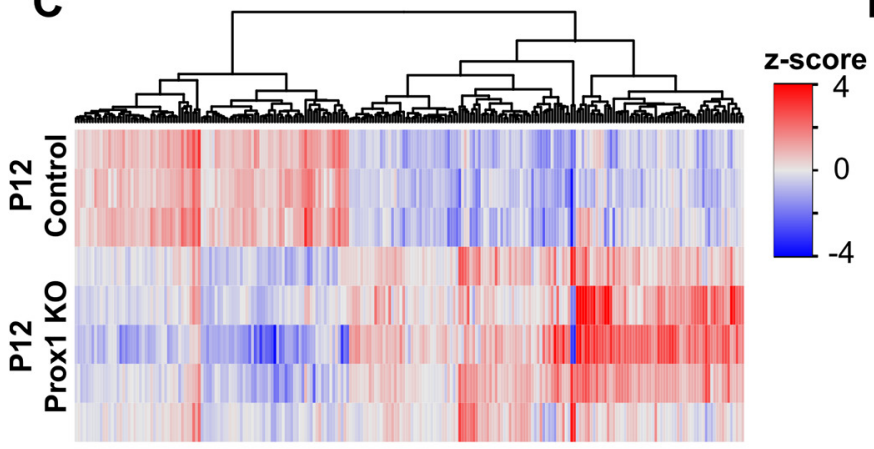

D

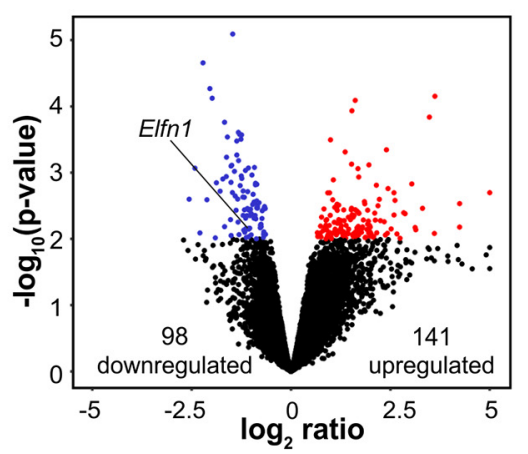

B

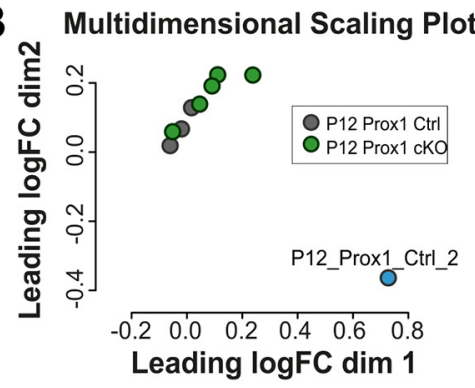

E
F

Biological Processes P12
eGFP positive

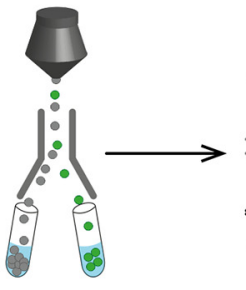

$\equiv \pm=$
RNAseq

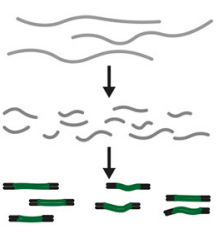

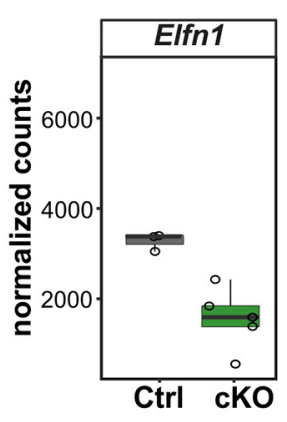

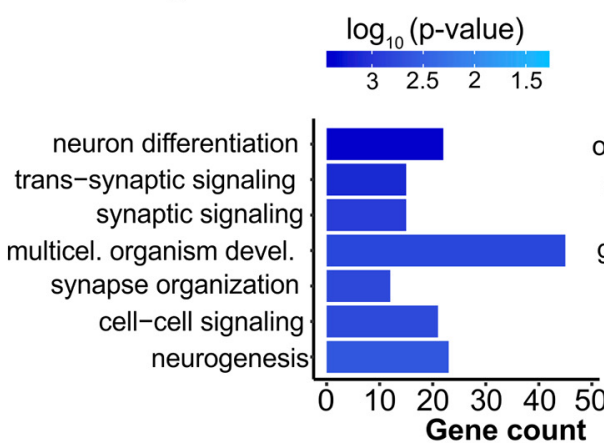

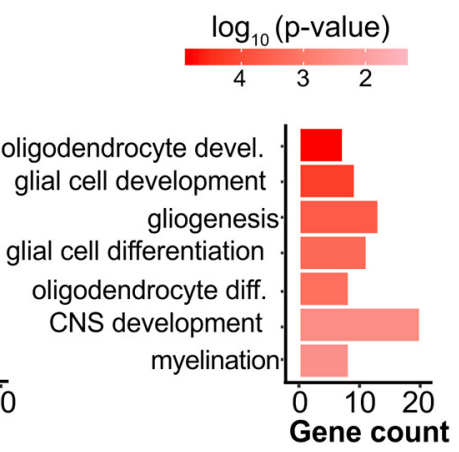

G

Figure 2. Postnatal removal of Prox1 from VIP cells leads to transcriptomic changes in Elfn1 and other synapse-related genes. $A$, Schematic of experimental workflow. VIPCre $x$ Prox1eGFP control and CKO cells were sorted using FACS at P8 and P12, and bulk RNA sequencing was performed. B, Multidimensional scaling plot of all the sequenced samples, showing the one control sample that did not meet the quality control criteria and was excluded from the analysis (blue point). $C$, Heat map showing the clustering according to function of upregulated (red) and downregulated (blue) genes at P12. D, Volcano plot highlighting the differentially expressed candidate genes at $P 12$. They were selected based on $\log 2$ ratio $\geq|0.5|$ and $p$ value $\leq 0.01$. Elfn 1 gene is highlighted. $\boldsymbol{E}$, Elfn 1 expression levels in P12 control and Prox1 cK0 VIP cells. $\boldsymbol{F}, \mathrm{G0}$ : Biological Processes enrichment analysis of the candidate genes at P12. Blue represents the downregulated genes. Red represents the upregulated gene. $\mathbf{G}, \mathrm{G0}$ : Cellular Components enrichment analysis of the candidate genes at P12. Blue represents the downregulated genes. The upregulated genes did not show any clustering in this $G 0$ term. See also Extended Data Figure 2-1.

whether Elfn 1 is continuously expressed into adulthood, we consulted published single-cell RNA sequencing data (Tasic et al., 2018) and found that, in the adult cortex, almost all VIP cells express Elfn1 (https://celltypes.brain-map.org/ rnaseq/mouse/v1-alm). Furthermore, this dataset also shows a persistent expression of Proxl, which suggests a requirement for both these genes in the maintenance of cell function beyond development.

\section{Reduction in Elfn1 expression recapitulates the Prox 1 cKO} phenotype in VIP multipolar cells

To test whether Proxl cKO alters the synaptic phenotype of multipolar VIP cells by reducing Elfn1 levels, we used a compound mouse line that labeled VIP neurons (VIPCre $\mathrm{x}$
tdTomato reporter; Ai14) in the background of a germline Elfn1 KO allele (Elfn1KO) (Fig. $3 A$ ). Since our RNA sequencing screen showed a relatively modest (twofold) reduction of Elfn1 transcript in Prox 1 cKO cortex (Fig. 2E), we chose to compare VIP tdTomato cells from heterozygous Elfn1KO (Elfn1 Het) animals to those from WT (control) littermates. We found that reducing Elfn1 expression does not affect the PPR significantly in VIP bipolar cells (control mean PPR: $1.15 \pm 0.14$; Elfn 1 Het mean PPR: $0.98 \pm 0.08$ ) (Fig. $3 B, C$ ). On the other hand, excitatory inputs onto VIP multipolar cells showed a notable reduction in the PPR when Elfn1 levels are reduced (control mean PPR: $1.30 \pm 0.13$; Elfn1 Het mean PPR: $0.88 \pm 0.08$ ) (Fig. 3D,E). Our results show that a decrease in Elfn1 
A

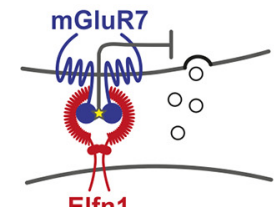

Genetic Removal of Elfn1

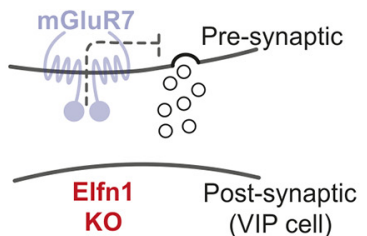

$\mathbf{F}$

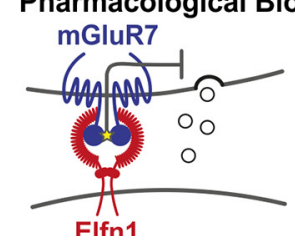

of Elfn1 action

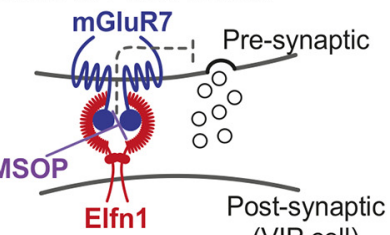

VIP Bipolar (VIPCre $x$ Ai14 x Elfn1KO)
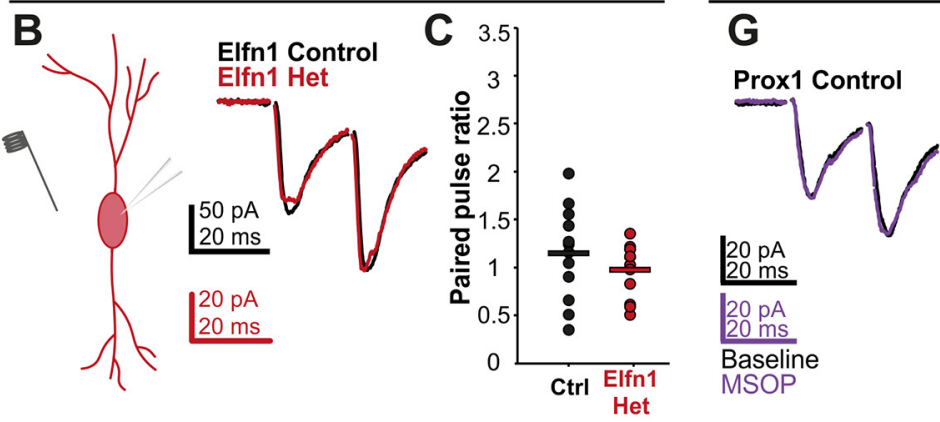

VIP Bipolar (VIPCre $x$ Prox1eGFP)

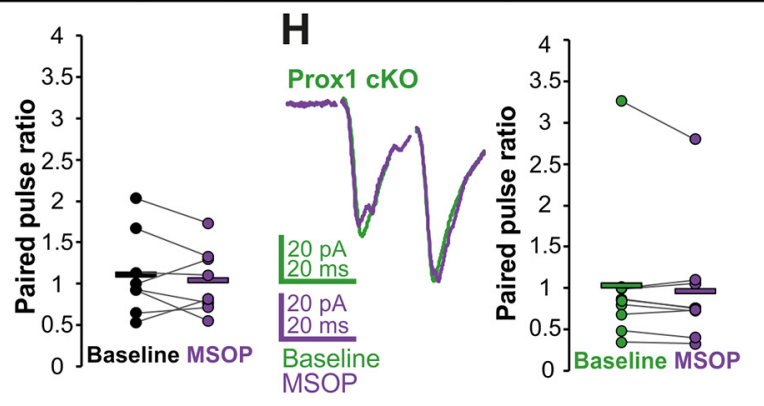

VIP Multipolar (VIPCre x Ai14 x Elfn1KO)
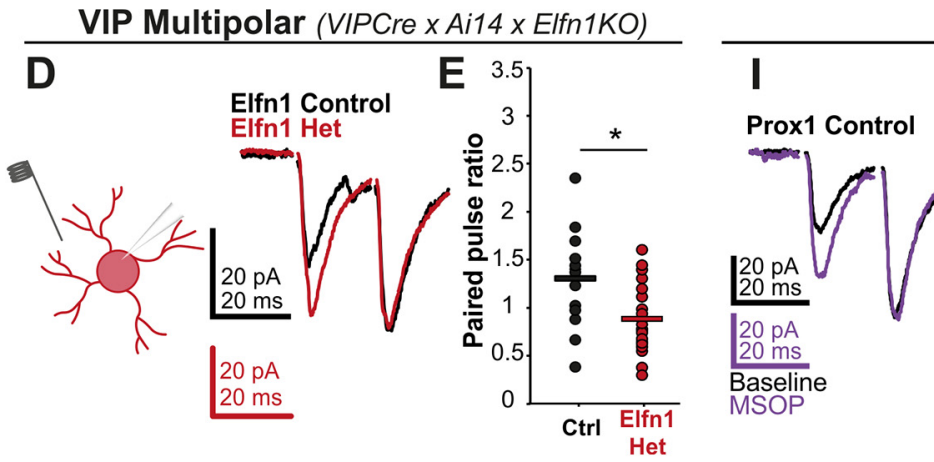

VIP Multipolar (VIPCre x Prox1eGFP)

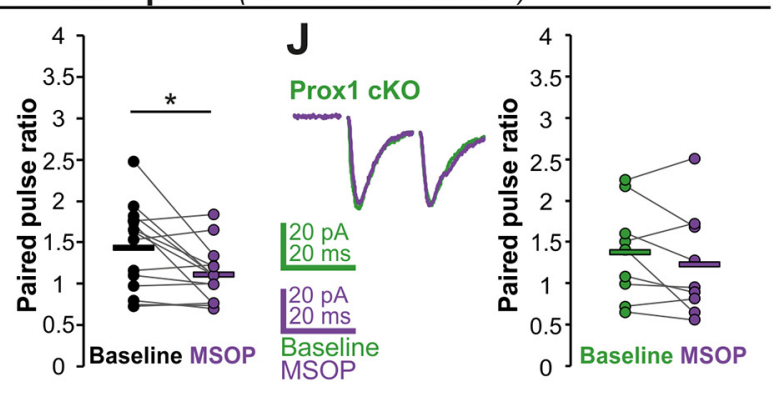

Figure 3. Elfn1 contributes to synaptic facilitation onto VIP multipolar, but not bipolar, cells. $A, A$ compound mouse line labeling VIP cells was crossed into the background of an Elfn 1 germline K0. The presence of Elfn1 decreases initial release probability of glutamate. The experimental and control groups were heterozygous (het) and WT (wt), respectively, for the Elfn1 germline removal. $\boldsymbol{B}, \boldsymbol{D}$, Left, Schematic representation of the evoked paired-pulse protocol, with the extracellular stimulating and intracellular recording electrodes shown. Right, Example of a pair of synaptic responses evoked onto VIP bipolar and multipolar cells after stimulation (scaled to second evoked response). $C$, All data points for PPR of Elfn1 Control $(n / N=12 / 4)$ and Het $(n / N=12 / 4)$ bipolar VIP cells $(p=0.3, t=1.06)$. $E$, All data points for PPR of Elfn1 Control $(n / N=14 / 6)$ and Het $(n / N=20 /$ 8) multipolar VIP cells $(p=0.008, t=2.84)$. Statistics: $t$ test. $\boldsymbol{F}$, Testing for the effect of MSOP, a presynaptic mGluR blocker and hence for Elfn 1 function, on evoked excitatory responses onto control (Het) and Prox1 cK0 cells. G-J, Left, Example of a pair of synaptic responses evoked onto VIP bipolar and multipolar cells after stimulation (scaled to second evoked response). Right, All data points for PPR are plotted. G, PPR of bipolar Prox1 Control cells $(n / N=8 / 8)$ plotted before and after the application of MSOP ( $p=0.5$, $t=0.74)$. $\boldsymbol{H}$, PPR of bipolar Prox1 cKO cells $(n / N=9 / 9)$ plotted before and after the application of MSOP $(p=0.2, t=1.26)$. $I$, PPR of multipolar Prox1 Control cells $(n / N=14 / 9)$ plotted before and after the application of MSOP $(p=0.02, t=2.74)$. J, PPR of multipolar Prox1 cKO cells $(n / N=9 / 8)$ plotted before and after the application of MSOP $(p=0.2$, $t=1.33$ ). Statistics: paired $t$ test. ${ }^{*} p<0.05$.

expression recapitulates the effect of Prox $1 \mathrm{cKO}$ in VIP multipolar cells and has no notable effect in bipolar cells. This finding suggests that Prox1 is important for initiating and/or maintaining the expression of Elfn1 in multipolar cells, which leads to facilitation of incoming excitatory synaptic responses.

Prox1-dependent engagement of Elfn1 in VIP cells

To directly test the relationship between Proxl and the expression of Elfn1 in multipolar and bipolar cells, we turned to a pharmacological agent, MSOP, which acts as an antagonist for presynaptic mGluRs (including mGluR7), which interact transsynaptically with Elfn1 (Fig. 3F) (Tomioka et al., 2014). Application of MSOP indirectly tests for the presence of Elfn1 in control and Proxl cKO VIP cells by blocking the constitutive suppression of synaptic release that Elfn1 induces through
mGluRs (Stachniak et al., 2019). We therefore evoked excitatory synaptic events onto the two VIP subtypes as described above and assessed changes in neurotransmitter release in response to MSOP. We found that MSOP did not affect synaptic facilitation onto control (PPR: $1.11 \pm 0.18$ at baseline vs $1.04 \pm 0.14$ in MSOP) or Prox 1 cKO VIP bipolar cells (PPR: $0.95 \pm 0.29$ at baseline vs $0.88 \pm 0.24$ in MSOP) (Fig. $3 G, H$ ). In contrast, MSOP markedly reduced the PPR of excitatory inputs onto control VIP multipolar cells (PPR: $1.43 \pm 0.14$ at baseline vs $1.11 \pm 0.09$ in MSOP) (Fig. 3I). However, the effect of MSOP was absent in the Proxl cKO VIP multipolar cells (PPR: $1.37 \pm 0.19$ at baseline vs $1.23 \pm 0.21$ in MSOP) (Fig. 3J). These pharmacological results suggest that Elfn1/mGluR7 complexes are only present in VIP multipolar synapses and that, on Prox1 removal, the levels of these complexes decrease enough to be functionally undetectable. To test this selective distribution 

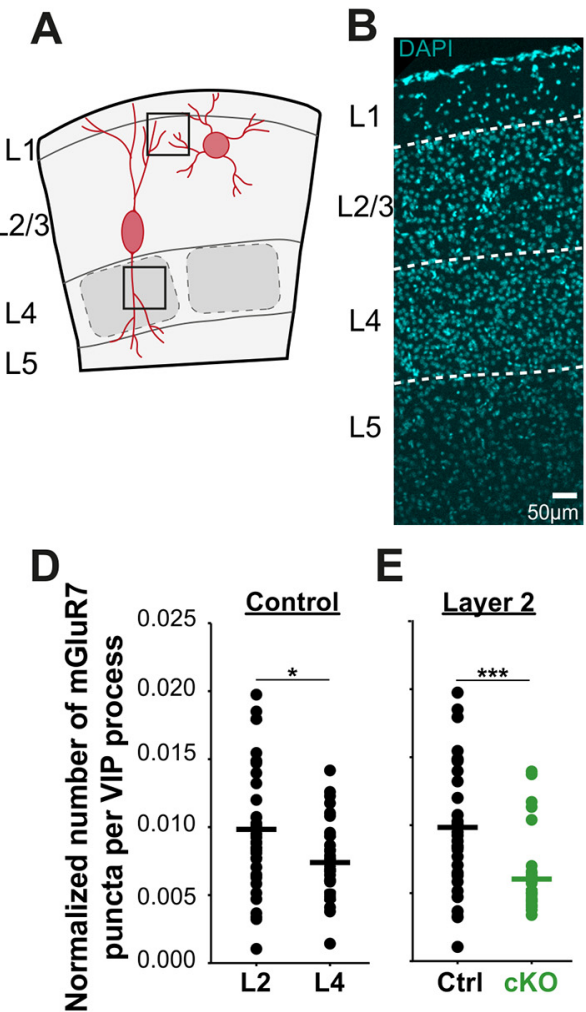

E
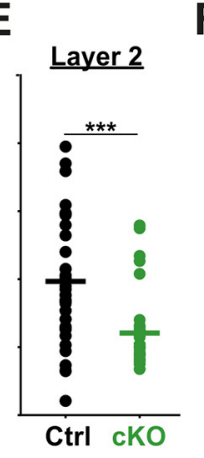

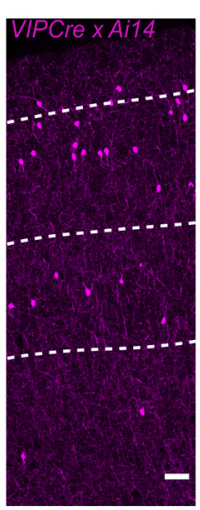

$\mathbf{F}$ Layer 4

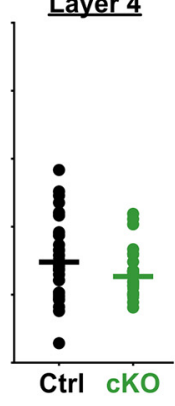

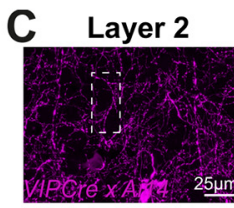
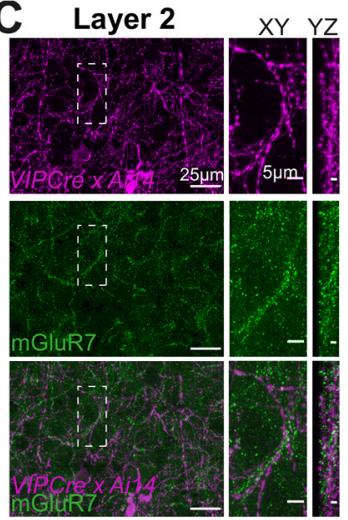

G
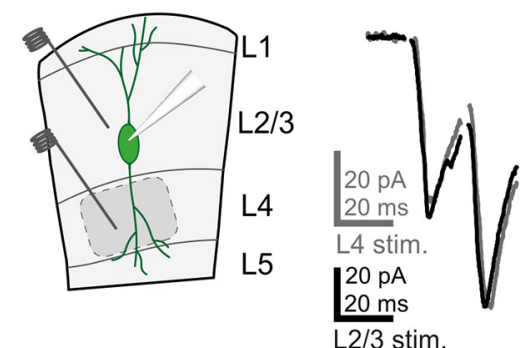

Prox1 Contro Bipolar
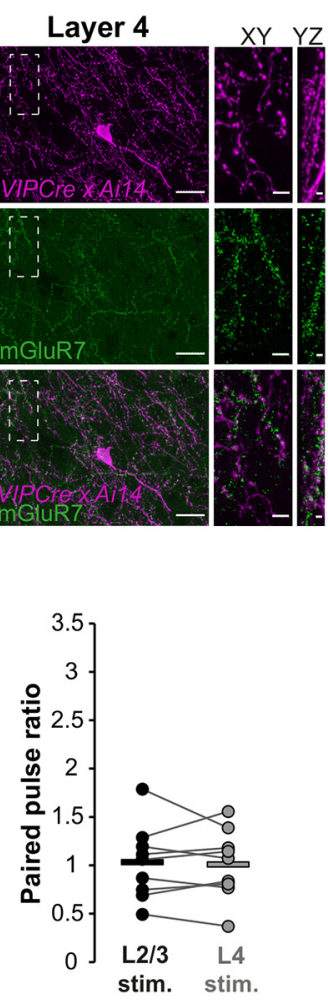

Figure 4. mGluR7 is preferentially recruited to L2 VIP processes. $A$, Schematic representation of the analysis area in L2/3 containing both multipolar and bipolar processes and in $\mathrm{L} 4$ containing only bipolar processes. $\boldsymbol{B}$, Segmentation of L1-4 in a coronal section of somatosensory cortex based on the DAPI channel. C, Example images of mGluR7 stainings in L2 and L4. Insets, Zoom-in images on the right showing mGluR7 puncta appositions on VIP processes in $x y$ and $y z$ directions. D, mGluR7 colocalization with VIP processes in L2 and L4 of Prox1 control tissue $(p=0.0178)(n=36$ images per layer, $N=3$ animals per genotype). $E$, mGluR7 colocalization with VIP processes in L2 of control $(n / N=36 / 3)$ and Prox1 $c K 0(n / N=32 / 3)$ tissue $(p=0.00014)$. $\boldsymbol{F}$, mGluR7 colocalization with VIP processes in $L 4$ of control $(n / N=36 / 3)$ and Prox 1 cK0 $(n / N=35 / 3)$ tissue $(p=0.063)$. Statistics: Mann-Whitney $U$ test. $G$, Left, Schematic illustration of stimulation paradigm for L2/3 bipolar neurons, a stimulation electrode is placed first in L2/3 and then in L4. Middle, Example of a pair of synaptic responses evoked in L2/3 and L4 in the same cell. Right, PPR of bipolar control cells when stimulated in $L 2 / 3$ and $L 4(p=0.8, t=0.20)$. Statistics: paired $t$ test. ${ }^{*} p<0.05 .{ }^{* * *} p<0.001$.

anatomically, we collected brains from control and Prox1 cKO animals (VIPCre $x$ Ail4 $x$ Proxlfl) at P21 and performed immunohistochemistry to label mGluR7-positive terminals. Presynaptic mGluR7 immunostaining is robust and requires the presence of postsynaptic Elfn1, thus providing an indirect measure of synaptic Elfn1 levels (Stachniak et al., 2019). Since bipolar cells have dendrites that extend over both L2/3 and L4, while multipolar cell dendrites tend to be restricted locally to $\mathrm{L} 2 / 3$, we reasoned that fluorescently labeled processes in L4 would stem predominantly from bipolar cells, while processes in L2 (upper L2/3) derive from both multipolar and bipolar neurons (Fig. 4A). We therefore quantified mGluR7 puncta colocalization with VIP processes in both upper L2/3 and L4 and compared between control and cKO conditions (Fig. 4B,C). First, we found that in control tissue L2 processes showed greater colocalization than those in L4 (Fig. 4D). Within L2, mGluR7 levels on VIP processes significantly decreased in Prox $1 \mathrm{cKO}$ compared with control condition (Fig. 4E). Since L4 processes (predominantly bipolar) showed no significant changes in mGluR7 levels on Prox1 removal (Fig. 4F), the large difference we see in L2 Prox 1 cKO is likely driven by changes on multipolar processes. To separately test whether bipolar cells show laminar differences in mGluR7 levels, we elicited synaptic responses onto bipolar cells by first stimulating in L2/3 and then stimulating in L4 and measuring the PPR. Within-cell analysis showed no differences in PPR between the two layers (L2/3 mean PPR $1.03 \pm 0.13$, L4 mean PPR: $1.01 \pm 0.12$ ) (Fig. $4 G$ ), suggesting that the laminar differences we observe are indeed because of differences in VIP subtype mGluR7 and by proxy Elfn1 levels.

\section{Prox1 regulates Elfn1 expression in all VIP INs}

Previous research has shown that both VIP bipolar and multipolar cells express Elfn1 in the adult cortex (Paul et al., 2017; Tasic et al., 2018). These transcriptomic data stand in apparent contradiction to the VIP multipolar cell-selective effects we observe in our Prox1 loss of function, Elfn1 downregulation, and pharmacological experiments. We therefore hypothesized that either Elfn 1 is expressed only in multipolar cells before P21 or that Prox1 regulates Elfn1 expression only in multipolar cells. To test these two possibilities, we collected and sectioned brains from Proxl control and cKO mice (Proxlfl x VIPCre x Ai14) at P12 and performed ISH for Elfn1 mRNA and CR mRNA to distinguish putative bipolar $\left(C R^{+}\right)$and multipolar $\left(C R^{-}\right)$VIP cells. We then obtained images of whole cortices and analyzed them using a customwritten MATLAB script (see Materials and Methods; Fig. $5 A-C)$. We first assessed the presence of Elfn $1 \mathrm{mRNA}$ in control VIP cells at P12 and found that, similarly to the adult cortex (Paul et al., 2017; Tasic et al., 2018), $C R^{+}$VIP cells express the gene at a higher level than $C R^{-}$VIP cells (Fig. 
5D). This finding negates the first hypothesis regarding an age-dependent selective expression of Elfn 1 in multipolar cells.

We subsequently compared Elfn1 and $C R$ mRNA levels between control and $\mathrm{CKO}$ tissue and found a clear reduction of both signals (Fig. 5E,F), which is in line with the RNAseq results that showed a downregulation of both genes (log2 ratio of -1.466 for $C R$ [Calb2] and -1.065 for Elfn1). Intriguingly, the reduction in Elfn 1 mRNA was seen for both $C R^{+}$(bipolar) VIP cells and $C R^{-}$VIP ones (most of which belong to the multipolar subtype) (Fig. 5G,H). These results suggest that Prox1 regulates Elfn1 in both subtypes of VIP cells, negating our second hypothesis.

\section{Elfn1 overexpression changes the synaptic phenotype of VIP bipolar cells}

A potential explanation for the discrepancy between Elfn1 transcript levels and function in VIP bipolar cells is that Elfn1 protein is largely absent from the synapse, either because the mRNA is not translated or because the protein is not transported there efficiently. We therefore examined the cell-autonomous effects of overexpressing Elfn1 protein in VIP bipolar neurons. We induced sparse virus-based overexpression of Elfn1 along with GFP in 3- to 5-week-old control bipolar cells and assessed the resulting short-term dynamics of synaptic responses, as well as the effect of MSOP on transfected and neighboring untransfected cells (Fig. 6A,B). As before, we found that, in untransfected VIP bipolar control cells, MSOP did not affect PPR (PPR: $0.73 \pm 0.13$ at baseline vs $0.72 \pm 0.13$ in MSOP) (Fig. $6 C)$. In contrast, when the bipolar cells overexpressed Elfn1, the PPR of excitatory inputs increased and MSOP significantly reduced it (PPR: $1.23 \pm 0.22$ at baseline vs $0.97 \pm 0.17$ in MSOP) (Fig. $6 D$ ). These results suggest that the absence of Elfn1 function in bipolar cells is indeed because of a lack of protein at the synapse, which can be overcome by overexpression of Elfn1.

In parallel experiments, we also assessed the overexpression of Elfn1 in 3- to 5-week-old multipolar VIP Prox1 cKO cells to see whether we can rescue the synaptic phenotype. In untransfected Prox 1 cKO multipolar cells, we found that MSOP did not affect PPR (PPR: $0.77 \pm 0.18$ at baseline vs $0.81 \pm 0.17$ in MSOP) (Fig. 6E), while it significantly reduced PPR in transfected neurons (PPR: $1.28 \pm 0.34$ at baseline vs $1.02 \pm 0.30$ in MSOP) (Fig. $6 F)$. Thus, by reintroducing Elfn 1 into Proxl cKO multipolar cells, the phenotype can be rescued to resemble the control condition.

\section{Discussion}

In the mammalian nervous system, the TF Proxl is known to regulate cell-cycle exit (Cid et al., 2010; Kaltezioti et al., 2010), cell fate determination (Iwano et al., 2012; Kaltezioti et al., 2014) of neural precursor cells, and neurite outgrowth (Kaltezioti et al., 2021). Prenatal removal of Proxl in embryonic caudal ganglionic eminence-derived cortical INs results in a failure of $\mathrm{CR}^{+}$VIP INs to migrate to the correct cortical layer, a significant decrease in their numbers, and a subsequent reduction in the excitatory synaptic input onto the remaining cells (Miyoshi et al., 2015). Postnatal removal of Proxl circumvents cell death and layer mis-targeting. Nevertheless, we find a continued requirement for the $\mathrm{TF}$ in the regulation of synaptic dynamics and the final specification of VIP subtypes. During the first two postnatal weeks, when VIP INs are embedding into their circuits, Prox1 upregulates genes related to synaptic processes and neuronal cell fate. Cell-specific expression of synapse-related genes, such as cell adhesion molecules, help guide the cell-cell interactions that ultimately determine the connectivity, morphologic, and electrophysiological characteristics of IN subtypes (Paul et al., 2017; Gouwens et al., 2020). One of these synapse-related genes is Elfn1, which codes for a trans-synaptic protein that produces synaptic facilitation of excitatory inputs in multipolar VIP cells. Although Prox 1 also regulates the expression of Elfn 1 transcript in bipolar VIP cells, they show negligible facilitation and Prox 1 removal does not impact bipolar PPR. Thus, by regulating VIP subtype-specific Elfn 1 engagement, the TF Prox1 promotes and maintains functional diversity in VIP IN subtypes.

Prox1-dependent upregulation of Elfn1 leads to excitatory synaptic facilitation specifically in VIP multipolar cells. In SST INs, this Elfn1-dependent facilitation of excitatory inputs prevents rapid recruitment, thereby creating their characteristic "delayed" firing property (Sylwestrak and Ghosh, 2012) and biasing responsiveness toward high-frequency activity $(>20 \mathrm{~Hz}$ ) (Pouille and Scanziani, 2004; Stachniak et al., 2019). The same mechanism would allow VIP multipolar cells to selectively tune to the high-frequency activity characteristic of corticocortical communication, whereas bipolar neurons would preferentially tune to lower excitation frequency (4-12 Hz) (Palmer et al., 2012; Luo et al., 2020). Within L2/3, VIP multipolar cells are often found close to the border with L1, whereas VIP bipolar cells usually are located closer to L4, which, in combination with their distinct dendritic architecture, could bias the amount of thalamocortical and corticocortical inputs received by the VIP subtypes (He et al., 2016; Sohn et al., 2016). Thus, by regulating Elfn1, Prox1 may prime multipolar neurons to coordinate intracortical communication within the superficial layers of the cortex. Interestingly, the SST INs do not express Prox1; therefore, our results not only identify a novel functional role for Elfn1 in VIP multipolar INs, but also reveal a novel regulatory pathway for $E l f n 1$ expression in general.

In contrast, short-term plasticity of excitatory inputs onto bipolar cells is unaffected by removal of Prox1, by decreased Elfn1 expression, or by the pharmacological blockade of its synaptic effects. These findings were very surprising given that our results and published transcriptomic data show high levels of Elfn1 mRNA expression in bipolar VIP cells. Thus, it appears that Elfn1 mRNA levels are decoupled from Elfn 1 function in this VIP subtype. Noting that mGluR7 protein levels were consistent with our observations of subtype-specific Elfn 1 function, we tested whether bipolar cells might have insufficient Elfn 1 protein levels, despite the high mRNA level. Overexpressing the Elfn1 protein in bipolar VIP cells by viral injection led to the recruitment of mGluR7 to the presynapse, confirming that Elfn1 levels in VIP INs are the primary determinant of the synaptic phenotype, as found previously (Sylwestrak and Ghosh, 2012). Hence, our results indicate that Proxl regulates the levels of Elfn1 mRNA in both VIP subtypes, but that there is an additional mechanism present in bipolar cells that prevents Elfn1 protein from being produced. Possible mechanisms could include 

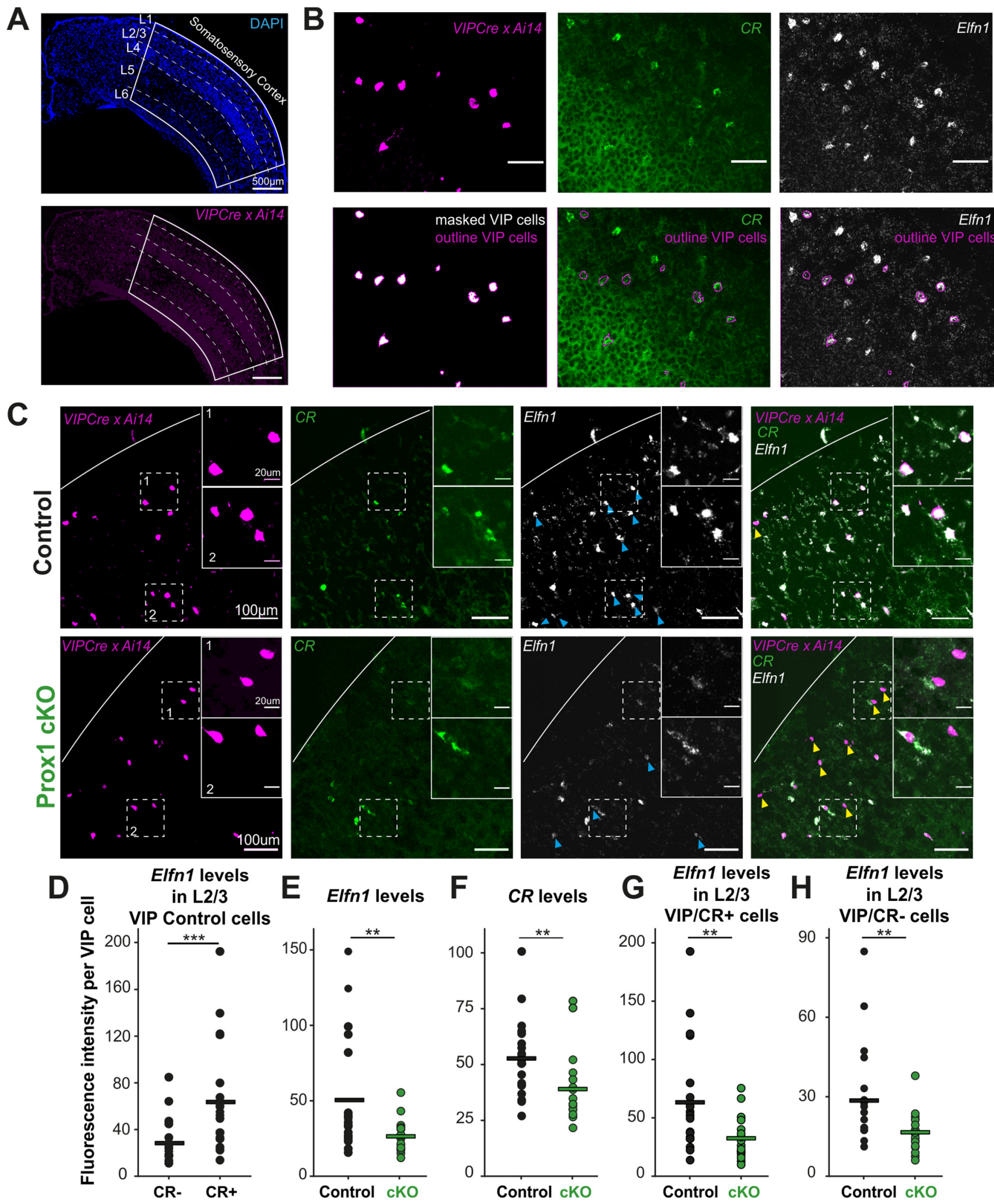

Figure 5. Prox1 regulates Elfn1 expression in all VIP INs. $\boldsymbol{A}$, Manual segmentation of somatosensory cortex and layers 1-6 based on the DAPI channel of coronal sections. $\boldsymbol{B}$, An example image showcasing the high-throughput analysis method. VIP cells were segmented, and $C R$ and Elfn 1 fluorescence levels were measured within the boundaries of the cell. $C$, Examples of Elfn1 and CR mRNA in situ (RNAscope) labeling in L2/3 tdTomato-positive VIP cells in control (top) and Prox1 cK0 (bottom) tissue at P12. Insets, Close-up of the dashed-line boxes, including $C R^{+}$and $C R^{-}$VIP cells. White parabolic line indicates the pia. Yellow arrows highlight VIP-positive Elfn1-negative cells. Blue arrows highlight Elfn1-positive VIP cells. There are also Elfn1-positive cells that are not VIP-positive likely corresponding to SST cells. D, Elfn 1 levels in VIP multipolar $\left(C R^{-}\right)$and bipolar $\left(C R^{+}\right)$cells in L2/3 of the somatosensory cortex $(p=0.001)$ ( $N=3$ animals, $n=19$ sections/images). $\boldsymbol{E}$, Elfn1 levels in VIP cells in all layers and cortical areas in control (N/n=3/19) and Prox1 cKO $(N / n=2 / 18)$ tissue $(p=0.004)$. $\boldsymbol{F}, C R$ levels in VIP cells in all layers and cortical areas in control $(N / n=3 / 19)$ and Prox1 $\mathrm{K}$ O tissue $(N / n=2 / 18)(p=0.004)$. $\mathbf{G}$, Elfn1 levels in VIP bipolar cells in L2/3 of the somatosensory cortex in control 
regulation of translation by microRNAs or alternative splicing. Indeed, it has been reported that Elfn1 can be regulated by the mirg miRNA cluster in an induced neuronal culture system (Whipple et al., 2020). Irrespective of the exact mechanism, our experiments show that viralinduced overexpression of Elfn1 can override any post-transcriptional regulatory process that limits Elfn 1 protein levels in bipolar cells. These findings suggest that subtype-specific post-transcriptional mechanisms contribute to VIP IN subtype diversity by reducing synaptic Elfn1 protein in bipolar cells.

In addition to the potential posttranscriptional regulatory mechanisms influencing Elfn1 protein levels, VIP subtype-specific protein-protein interactions across the synapse may also exist. Indeed, Elfn 1 has been shown to interact with multiple mGluR subtypes, and heterodimerization of mGluR7 with other mGluRs changes synaptic facilitation and MSOP responsiveness (Kammermeier, 2015; Levitz et al., 2016; Dunn et al., 2018). Homodimerization of mGluR7 produces constitutive activation of the receptor, conferring facilitating properties and MSOP sensitivity onto synapses (Kammermeier, 2015; Stachniak et al., 2019). In contrast, heterodimerization (e.g., mGluR7/mGluR2 or mGluR7/ mGluR4) increases glutamate responsiveness, thus producing synaptic depression, and also leads to the loss of MSOP responsiveness (Kammermeier, 2015; Levitz et al., 2016; Stachniak et al., 2019). Thus in multipolar VIP synapses, higher levels of Elfn1 protein may trigger a cellselective switch in PPR by promoting formation of mGluR7 homodimers over heterodimers.

The final specification of neurons is a process that takes place long after their birth, as they start incorporating themselves into the resident circuit. It is during this time of establishing connections that the needs of the circuit, as defined by those of the animal, can instruct a neuron toward its mature state, which includes the proper construction and function of inputs and outputs. For inhibitory INs, these input/output specificities vary considerably, not only between cardinal IN classes, but also between the subtypes within a class (Huang and Paul, 2019). We find that postnatal Prox1 expression in VIP cells diversifies the dynamics of incoming excitation onto the multipolar versus the

$(N / n=3 / 19)$ and Prox1 cKO $(N / n=2 / 18)$ tissue $(p=0.004)$. $\boldsymbol{H}$, Elfn1 levels in VIP multipolar cells in $L 2 / 3$ of the somatosensory cortex in control $(N / n=3 / 19)$ and Prox1 CKO (N/ $n=2 / 18)$ tissue $(p=0.006)$. Statistics: Mann-Whitney $U$ test. ${ }^{* *} p<0.01 .{ }^{* *} p<0.001$. bipolar subtypes. Our rescue and overexpression experiments in late juvenile/young adult mice (postnatal weeks 35) show that Elfn 1 expression is necessary to maintain this synaptic diversity. Since Prox1 is present in VIP INs of adult mice, it is likely that the continued presence of Proxl is required for maintaining the synaptic phenotype we have uncovered. Our data provide evidence that the continuous expression of the TF Proxl is necessary for the final specification of bipolar and multipolar VIP cells, allowing them to acquire diverging roles within the adult cortical network.

\section{References}

Batista-Brito R, Vinck M, Ferguson KA, Chang JT, Laubender D, Lur G, Mossner JM, Hernandez VG, Ramakrishnan C, Deisseroth K, Higley MJ, Cardin JA (2017) Developmental dysfunction of VIP interneurons impairs cortical circuits. Neuron 95:884-895.e9. 
Bayraktar T, Staiger JF, Acsady L, Cozzari C, Freund TF, Zilles K (1997) Colocalization of vasoactive intestinal polypeptide, $\gamma$-aminobutyric acid and choline acetyltransferase in neocortical interneurons of the adult rat. Brain Res 757:209-217.

Bayraktar T, Welker E, Freund TF, Zilles K, Staiger JF (2000) Neurons immunoreactive for vasoactive intestinal polypeptide in the rat primary somatosensory cortex: morphology and spatial relationship to barrelrelated columns. J Comp Neurol 420:291-304.

Bolger AM, Lohse M, Usadel B (2014) Trimmomatic: a flexible trimmer for Illumina sequence data. Bioinformatics 30:2114-2120.

Caputi A, Rozov A, Blatow M, Monyer H (2009) Two calretinin-positive GABAergic cell types in layer $2 / 3$ of the mouse neocortex provide different forms of inhibition. Cereb Cortex 19:1345-1359.

Cauli B, Zhou X, Tricoire L, Toussay X, Staiger JF (2014) Revisiting enigmatic cortical calretinin-expressing interneurons. Front Neuroanat 8:52.

Cid E, Santos-Ledo A, Parrilla-Monge M, Lillo C, Arévalo RR, Lara JM, Aijón J, Velasco A (2010) Prox1 expression in rod precursors and Müller cells. Exp Eye Res 90:267-276.

De La Rossa A, Bellone C, Golding B, Vitali I, Moss J, Toni N, Lüscher C, Jabaudon D (2013) In vivo reprogramming of circuit connectivity in postmitotic neocortical neurons. Nat Neurosci 16:193-200.

De Marco García NV, Karayannis T, Fishell G (2011) Neuronal activity is required for the development of specific cortical interneuron subtypes. Nature 472:351-355.

De Marco García NV, Priya R, Tuncdemir SN, Fishell G, Karayannis T, De Marco GN, Priya R, Tuncdemir SN, Fishell G, Karayannis T (2015) Sensory inputs control the integration of neurogliaform interneurons into cortical circuits. Nat Neurosci 18:393-401.

Dobin A, Davis CA, Schlesinger F, Drenkow J, Zaleski C, Jha S, Batut P, Chaisson M, Gingeras TR (2013) STAR: ultrafast universal RNA-seq aligner. Bioinformatics 29:15-21.

Dunn HA, Patil DN, Cao Y, Orlandi C, Martemyanov KA (2018) Synaptic adhesion protein ELFN1 is a selective allosteric modulator of group III metabotropic glutamate receptors in trans. Proc Natl Acad Sci USA 115:5022-5027.

Favuzzi E, Deogracias R, Marques-Smith A, Maeso P, Jezequel J, ExpositoAlonso D, Balia M, Kroon T, Hinojosa AJ, Maraver EF, Rico B (2019) Neurodevelopment: distinct molecular programs regulate synapse specificity in cortical inhibitory circuits. Science 363:413-417.

Gouwens NW, Sorensen SA, Baftizadeh F, Budzillo A, Lee BR, Jarsky T, Alfiler L, Baker K, Barkan E, Berry K, Bertagnolli D, Bickley K, Bomben J, Braun T, Brouner K, Casper T, Crichton K, Daigle TL, Dalley R, de Frates RA, et al. (2020) Integrated morphoelectric and transcriptomic classification of cortical GABAergic cells. Cell 183:935-953.e19.

Harvey NL, Srinivasan RS, Dillard ME, Johnson NC, Witte MH, Boyd K, Sleeman MW, Oliver G (2005) Lymphatic vascular defects promoted by Prox1 haploinsufficiency cause adult-onset obesity. Nat Genet 37:10721081.

He M, Tucciarone J, Lee SH, Nigro MJ, Kim Y, Levine JM, Kelly SM, Krugikov I, Wu P, Chen Y, Gong L, Hou Y, Osten P, Rudy B, Huang ZJ (2016) Strategies and tools for combinatorial targeting of GABAergic neurons in mouse cerebral cortex. Neuron 91:1228-1243.

Hempel CM, Sugino K, Nelson SB (2007) A manual method for the purification of fluorescently labeled neurons from the mammalian brain. Nat Protoc 2:2924-2929.

Huang ZJ, Paul A (2019) The diversity of GABAergic neurons and neural communication elements. Nat Rev Neurosci 20:563-572.

Iwano T, Masuda A, Kiyonari H, Enomoto H, Matsuzaki F (2012) Prox1 postmitotically defines dentate gyrus cells by specifying granule cell identity over CA3 pyramidal cell fate in the hippocampus. Development 139:3051-3062.

Kaltezioti V, Kouroupi G, Oikonomaki M, Mantouvalou E, Stergiopoulos A, Charonis A, Rohrer H, Matsas R, Politis PK (2010) Proxl regulates the Notch1-mediated inhibition of neurogenesis. PLoS Biol 8:e1000565.

Kaltezioti V, Antoniou D, Stergiopoulos A, Rozani I, Rohrer H, Politis PK (2014) Proxl regulates Olig2 expression to modulate binary fate decisions in spinal cord neurons. J Neurosci 34:15816-15831.

Kaltezioti V, Foskolou IP, Lavigne MD, Ninou E, Tsampoula M, Fousteri M, Margarity M, Politis PK (2021) Prox1 inhibits neurite outgrowth during central nervous system development. Cell Mol Life Sci 78:3443-3465.
Kammermeier PJ (2015) Constitutive activity of metabotropic glutamate receptor 7. BMC Neurosci 16:17.

Kass M, Witkin A, Terzopoulos D (1988) Snakes: active contour models. Int J Comput Vision 1:321-331.

Kubota Y, Shigematsu N, Karube F, Sekigawa A, Kato S, Yamaguchi N, Hirai Y, Morishima M, Kawaguchi Y (2011) Selective coexpression of multiple chemical markers defines discrete populations of neocortical GABAergic neurons. Cereb Cortex 21:1803-1817.

Levitz J, Habrian C, Bharill S, Fu Z, Vafabakhsh R, Isacoff EY (2016) Mechanism of assembly and cooperativity of homomeric and heteromeric metabotropic glutamate receptors. Neuron 92:143159.

Liao Y, Smyth GK, Shi W (2013) The Subread aligner: fast, accurate and scalable read mapping by seed-and-vote. Nucleic Acids Res 41: e108.

Luo X, Guet-McCreight A, Villette V, Francavilla R, Marino B, Chamberland S, Skinner FK, Topolnik L (2020) Synaptic mechanisms underlying the network state-dependent recruitment of VIP-expressing interneurons in the CA1 hippocampus. Cereb Cortex 30:3667-3685.

Madisen L, Zwingman TA, Sunkin SM, Oh SW, Zariwala HA, Gu H, Ng LL, Palmiter RD, Hawrylycz MJ, Jones AR, Lein ES, Zeng H (2010) A robust and high-throughput Cre reporting and characterization system for the whole mouse brain. Nat Neurosci 13:133-140.

Mayer C, Hafemeister C, Bandler RC, Machold R, Batista Brito R, Jaglin X, Allaway K, Butler A, Fishell G, Satija R (2018) Developmental diversification of cortical inhibitory interneurons. Nature 555:457-462.

Mi D, Li Z, Lim L, Li M, Moissidis M, Yang Y, Gao T, Hu TX, Pratt T, Price DJ, Sestan N, Marín O (2018) Early emergence of cortical interneuron diversity in the mouse embryo. Science 360:81-85.

Miyoshi G, Young A, Petros T, Karayannis T, McKenzie Chang M, Lavado A, Iwano T, Nakajima M, Taniguchi H, Huang ZJ, Heintz N, Oliver G, Matsuzaki F, Machold RP, Fishell G (2015) Prox1 regulates the subtypespecific development of caudal ganglionic eminence-derived GABAergic cortical interneurons. J Neurosci 35:12869-12889.

Mossner JM, Batista-Brito R, Pant R, Cardin JA (2020) Developmental loss of $\mathrm{MeCP} 2$ from VIP interneurons impairs cortical function and behavior. Elife 9:e55639.

Palmer L, Murayama M, Larkum M (2012) Inhibitory regulation of dendritic activity in vivo. Front Neural Circuits 6:26

Pan NC, Fang A, Shen C, Sun L, Wu Q, Wang X (2018) Early excitatory activity-dependent maturation of somatostatin interneurons in cortical layer 2/3 of mice. Cereb Cortex 29:4107-4118.

Paul A, Crow M, Raudales R, He M, Gillis J, Huang ZJ (2017) Transcriptional architecture of synaptic communication delineates GABAergic neuron identity. Cell 171:522-539.e20.

Picelli S, Björklund ÅK, Faridani OR, Sagasser S, Winberg G, Sandberg R (2013) Smart-seq2 for sensitive full-length transcriptome profiling in single cells. Nat Methods 10:1096-1098.

Porter JT, Cauli B, Staiger JF, Lambolez B, Rossier J, Audinat E (1998) Properties of bipolar VIPergic interneurons and their excitation by pyramidal neurons in the rat neocortex. Eur J Neurosci 10:36173628 .

Pouille F, Scanziani M (2004) Routing of spike series by dynamic circuits in the hippocampus. Nature 429:717- 723.

Prönneke A, Scheuer B, Wagener RJ, Möck M, Witte M, Staiger JF (2015) Characterizing VIP neurons in the barrel cortex of VIPcre/ tdTomato mice reveals layer-specific differences. Cereb Cortex 25:4854-4868.

Raudvere U, Kolberg L, Kuzmin I, Arak T, Adler P, Peterson H, Vilo J (2019) g: profiler: a web server for functional enrichment analysis and conversions of gene lists (2019 update). Nucleic Acids Res 47: W191-W198.

Robinson MD, McCarthy DJ, Smyth GK (2010) edgeR: a Bioconductor package for differential expression analysis of digital gene expression data. Bioinformatics 26:139-140.

Rubin AN, Kessaris N (2013) PROX1: a lineage tracer for cortical interneurons originating in the lateral/caudal ganglionic eminence and preoptic area. PLoS One 8:e77339.

Sohn J, Okamoto S, Kataoka N, Kaneko T, Nakamura K, Hioki H (2016) Differential inputs to the perisomatic and distal-dendritic compartments 
of VIP-positive neurons in layer $2 / 3$ of the mouse barrel cortex. Front Neuroanat 10:124.

Stachniak TJ, Sylwestrak EL, Scheiffele P, Hall BJ, Ghosh A (2019) Elfn1-induced constitutive activation of mGluR7 determines frequencydependent recruitment of somatostatin interneurons. J Neurosci 39: 4461-4474.

Sylwestrak EL, Ghosh A (2012) Elfn1 regulates target-specific release probability at CA1-interneuron synapses. Science 338:536-540.

Taniguchi H, He M, Wu P, Kim S, Paik R, Sugino K, Kvitsiani D, Kvitsani D, Fu Y, Lu J, Lin Y, Miyoshi G, Shima Y, Fishell G, Nelson SB, Huang ZJ (2011) A resource of Cre driver lines for genetic targeting of GABAergic neurons in cerebral cortex. Neuron 71:995-1013
Tasic B, Yao Z, Graybuck LT, Smith KA, Nguyen TN, Bertagnolli D, Goldy J, Garren E, Economo MN, Viswanathan S, Penn O, Bakken T, Menon V, Miller J, Fong O, Hirokawa KE, Lathia K, Rimorin C, Tieu M, Larsen R, et al. (2018) Shared and distinct transcriptomic cell types across neocortical areas. Nature 563:72-78.

Tomioka NH, Yasuda $\mathrm{H}$, Miyamoto $\mathrm{H}$, Hatayama $\mathrm{M}$, Morimura $\mathrm{N}$, Matsumoto Y, Suzuki T, Odagawa M, Odaka YS, Iwayama Y, Won Um J, Ko J, Inoue Y, Kaneko S, Hirose S, Yamada K, Yoshikawa T, Yamakawa $\mathrm{K}$, Aruga J (2014) Elfn1 recruits presynaptic mGluR7 in trans and its loss results in seizures. Nat Commun 5:4501.

Whipple AJ, Breton-Provencher V, Jacobs HN, Chitta UK, Sur M, Sharp PA (2020) Imprinted maternally expressed microRNAs antagonize paternally driven gene programs in neurons. Mol Cell 78:85-95.e8. 